\title{
Minimization of the electric energy in systems using ultra-high density magnetic storage
}

\author{
PAKAM Tchilabalo ${ }^{1}$, A. Adanlété Adjanoh ${ }^{1}$ \\ ${ }^{1}$ Laboratoire Matériaux et Energie Renouvelable et Environnement (LaMERE), University of Kara, Togo.
}

\begin{abstract}
We present an optimization of the thickness of the magnetic layers that serve to record the information of the daily need in order to minimize the useful electrical energy. The study provides details on the energy activation and distribution of the energy barriers in the samples of thickness $t_{C_{o}}=0.7,0.8$ and $1 \mathrm{~nm}$. We find that distribution of the energy barriers $\mathrm{E}_{\mathrm{a}}$, its distribution width $\sigma_{w}$, the real activation field $\mu_{0} \mathrm{H}_{\mathrm{r}}$ are lowest in the sample of thickness $t_{C_{o}}=1 \mathrm{~nm}$, hence this sample allows to use less electrical energy for information recording.
\end{abstract}

Keywords: Width of distribution of the energy barriers, information recorging, electrical energy minimization.

\section{Introduction}

The evolution of the digital economy and the use of ferromagnetic materials in ultra high density information storage has given rise to interest in these materials in recent decades for researchers. Research on these materials is oriented either towards the understanding of very fundamental mechanisms, or towards important perspectives for applications such as ultra high density storage [1,2]. In fact, the writing of elementary information is traditionally done by applying a magnetic field pulse [3]. The field and energy required to create a first magnetization reversal are called the activation field and activation energy, respectively. But the activation energy is not single in a real sample. In this case the simple activation energy is replaced by a distribution of energy barriers[10]. The lower the distribution of energy barriers, the less electrical energy is needed for the storage of information. Therefore, perfect control and optimization of these parameters controlling the distribution of energy barriers in order to reduce this energy as much as possible, is essential in order to minimize the electrical energy required for this effect. Some works has been devoted to the activation energy $[3,4,5,6]$ but did not discuss the effect of the thickness on the distruibution and the width of the energy barriers in the samples. This paper has the particularity of showing that the thickness of the magnetic layers have an influence on the width of the distribution of energy barrier, hence on electrical energy.

\section{Material and Method}

\section{Sample and Structural Characterizations}

$\mathrm{Si}(100)$ substrate is beforehand cleaned by ultrasounds in an acetone bath. After the cleaning, this substrates is thermally oxidized in a furnace at $1200^{\circ} \mathrm{C}$ during 2 hours. This time is sufficient for the formation of an oxide layer on the silicon surface substrate.

$\mathrm{Au} / \mathrm{Co} / \mathrm{Au}$ films were prepared by electron beam evaporation in an ultrahigh vacuum chamber, with a base pressure about of $10^{-9} \mathrm{Torr}$ and approximately $10^{-8} \mathrm{Torr}$ during 
deposition on $\mathrm{SiO} 2$, at room temperature. On Au film, cobalt layers with thicknesses $\left(t_{C_{o}}\right): 1$, 0.8 and $0.7 \mathrm{~nm}$ are then deposited [11]. At finally a second Au layer with a thickness about of $5 \mathrm{~nm}$ is deposited on top of the cobalt layers. The (111) texture of the Au buffer layer suggests, in each case, a possible epitaxial growth of the cobalt layer with the Hexagonal Close-Packed (0001) structure [7,8,9].

\section{Megnetic investigation}

Magnetic hysteresis loops, at a field sweep rate of $\frac{d \mu_{0} H}{d t}=1.2 \mathrm{mT}$, were recorded at room temperature (RT) by polar magneto-optical Kerr effect magnetometry (PMOKE) using a HeNe laser $(\lambda=633 \mathrm{~nm})$. On the hysteresis loops we measured the coercive fields $\mu_{0} H_{c}$ and the nucleation fields $\mu_{0} H_{n}$. Table 1 . shows magnetic quasi statistic parameters deduced from the hysteresis loops of the three samples [4].

Table 1. Data obtained from the quasi-static characterizations

\begin{tabular}{|l|c|c|c|}
\hline $\boldsymbol{t}_{\boldsymbol{C}_{\boldsymbol{o}}}(\mathrm{nm})$ & $\mathbf{0 . 7}$ & $\mathbf{0 . 8}$ & $\mathbf{1}$ \\
\hline $\boldsymbol{\mu}_{\mathbf{0}} \boldsymbol{H}_{\boldsymbol{c}}(\mathrm{mT})$ & 31.60 & 29.20 & 26.50 \\
\hline$\mu_{\mathbf{0}} H_{n}(\mathrm{mT})$ & 27.4 & 24.9 & 23 \\
\hline
\end{tabular}

\section{Magnetization Reversal}

\section{- Average activation energy}

The energy needed to reverse magnetization can be expressed in the following way $[3,5]$ :

$$
\mathrm{W}(\mathrm{H})=\bar{E}_{a}-\mathrm{M}_{\mathrm{s}} \mathrm{V}_{\mathrm{B}}\left(\mu_{0} \mathrm{H}\right)
$$

and the time $t_{1 / 2}$ corresponding to the time at the end of which the sample is demagnetized, is expressed following Arrhenius law :

$$
\mathrm{t}_{1 / 2}=\mathrm{t}_{0} \exp \left(\frac{\bar{E}_{a}-\mathrm{M}_{\mathrm{s}} \mathrm{V}_{\mathrm{B}}\left(\mu_{0} \mathrm{H}\right)}{\mathrm{K}_{\mathrm{B}} \mathrm{T}}\right)
$$

Where $\bar{E}_{a}$ is average activation energy at zero field, thermal energy required to initiate the magnetization reversal in the absence of the field, $M_{S}$ is the saturation magnetization and $V_{B}$ is the Barkhausen volume (the magnetization volume that reverses during a single activation event). The fitting of expérimental dots of $t_{1 / 2}$ vs $\mu_{0} \mathrm{H}$ and their ajustement by eq (2) allows to have the values of $t_{0}, \bar{E}_{a}$ and $\mathrm{M}_{\mathrm{s}} \mathrm{V}_{\mathrm{B}}$ for differents samples represented on figure 1. And in table 2 [4]. 


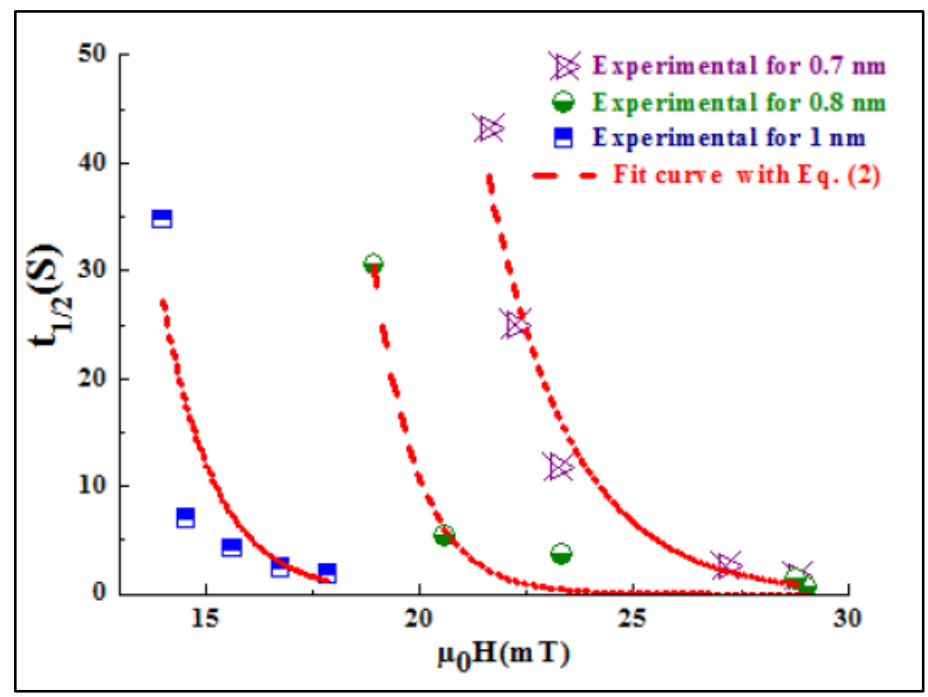

Figure 1. $t_{1 / 2}$ depending on $\mu_{0} \mathrm{H}$ and it fitting by Arrhenius-Néel law Eq. (2), for the three samples

Table 2. Data from the fitting byArrhenius-Néel law

\begin{tabular}{|c|c|c|c|}
\hline $\mathbf{t}_{\mathbf{C} \mathbf{0}}(\mathbf{n m})$ & $\mathbf{0 . 7}$ & $\mathbf{0 . 8}$ & $\mathbf{1}$ \\
\hline $\mathbf{t}_{\mathbf{0}}(\boldsymbol{s})$ & $10^{4}$ & $10^{4}$ & $10^{4}$ \\
\hline$\overline{\boldsymbol{E}}_{\boldsymbol{a}}(\boldsymbol{m e V})$ & 146.12 & 329.2 & 138.4 \\
\hline $\mathbf{M}_{\mathbf{s}} \mathbf{V}_{\mathbf{B}}\left(\mathbf{1 0}^{-\mathbf{2 1}} \mathbf{J} / \boldsymbol{m} \boldsymbol{T}\right)$ & 2.15 & 4.05 & 3.35 \\
\hline
\end{tabular}

- Width of the distribution of the energy barriers

According to the work of A. Adanlété Adjanoh, R. Belhi [10,11], the weakness of $\bar{E}_{a}$ confirms the fact that the magnetization reversal in thises samples is mainly done by domain wall motion in the sample. In a real sample the magnetic domains have a dendritic structure as shown in image of fig. 3 . This indicates that an activation energy would not be single in the sample. In this case the simple energy barrier is replaced by a distribution of energy barriers, characterized by a width $\sigma_{w}$. Bruno et al. [3] showed that if one assumes a square distribution of the energy barriers, the maximum slope of reduced magnetization $m(t)=(M(t)+M s) / 2 M s$ represented as a function of $\ln (t)$ is inversely proportional to the width $\sigma_{w}$ of the distribution of the activation energy barriers:

$$
m(t)=-\frac{K_{B} T * \ln t}{\sigma_{w}}
$$

The relaxation curves $(t)$ vs $(t)$ and their fittings using Eq.(3) for the layer of $1 \mathrm{~nm}$ are presented on fig. 2 . 


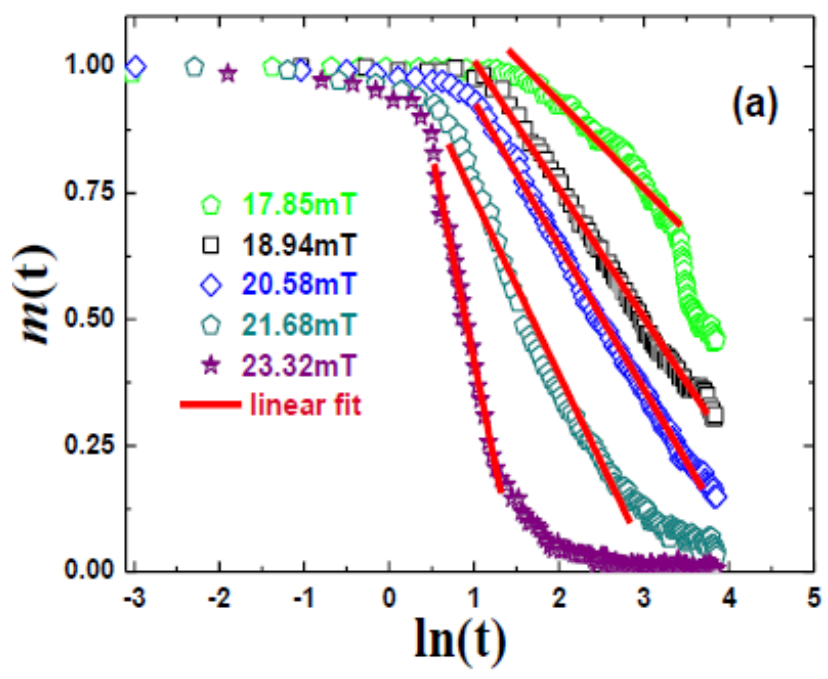

\section{Results and discussions}

\section{Width of the distribution of the energy barriers}

By analyzing the shapes of the curves and their fittings we can write this :

$$
\begin{aligned}
& \text { For } \ln t \epsilon]-\infty, 0] ; m(t)=c t e=1 \\
& \text { For } \ln t \epsilon[0, b] ; m(t)=\alpha \ln t+\beta
\end{aligned}
$$

With $\alpha$ and $\mathrm{b}$ values depending on the applied field and $\beta$ a constant.

At the start of the reversal, the magnetization keeps its saturation value for a period of time (Eq.1), after this demagnetization is almost linear with a negative slope which depends on the field $\left(\mu_{0} \mathrm{H}\right)$ applied.

Using the linear fittings and Eq.(5), We determine the value of $\beta=1 . \sigma_{w}$ Depends on applied field [13]. Then $\alpha$ Inversely proportional to $\sigma_{w}$. Let's take $\alpha=-\frac{K_{B} T}{\sigma_{w}}$, a negative slope due to decreases in the lines; we can then rewrite the eq. (5) as following:

$$
m(t)=-\frac{K_{B} T \ln t}{\sigma_{w}}+1
$$

From this relation we can determine the demagnetization time $t_{1 / 2}$ such that

$$
\frac{1}{2}=-\frac{K_{B} T \ln \left(t_{1 / 2}\right)}{\sigma_{w}}+1
$$


Which implies :

$$
t_{1 / 2}=\exp \left(\frac{\frac{1}{2} \sigma_{w}}{K_{B} T}\right)
$$

Now let us recall the expression of $t_{1 / 2}$ that follows Arrhenius' law in Eq. (1):

$$
\mathrm{t}_{1 / 2}=\mathrm{t}_{0} \exp \left(\frac{\bar{E}_{a}-\mathrm{M}_{\mathrm{S}} \mathrm{V}_{\mathrm{B}}\left(\mu_{0} \mathrm{H}\right)}{\mathrm{K}_{\mathrm{B}} \mathrm{T}}\right)
$$

Whith $\bar{E}_{a}$ the average of activation energy.

By equalizing the two relations (1) and (7) we end up with the expression for the width of the energy barrier:

$$
\sigma_{w}=\sigma_{w_{0}}-2 \mu_{0} M_{S} V_{B} H
$$

With

$$
\sigma_{w_{0}}=2\left(\bar{E}_{a}+K_{B} T \ln t_{0}\right)
$$

corresponding to the width of the distribution of the energy barriers at zero field.

This relation (Eq.(8)) shows that $\sigma_{w}$ decreases as the applied field increases, which is in perfect agreement with the experimental measurement of $\sigma_{w}$ in the sample of $1 \mathrm{~nm}$ thick. With Eq. (7), we can calculate the value of $\sigma_{w_{0}}$ for the three samples. We notice that by using $t_{0}=10^{4}$ we get high values of $\sigma_{w_{0}}$ compared to those measured experimentally [10].

This leads us to think about the real value of $t_{0}$ in a real sample. Taking into account the dendritic form of the domains observed experimentally, the magnetization reversal curves for theses samples can be described by the compressed exponential form [11]:

$$
m(t)=\exp \left[-\left(\frac{R t}{\tau(k)}\right)^{\beta}\right]
$$

Where $\mathrm{R}$ is the nucleation rate and $\tau(k)$ a parameter inversely proportional to $k$ which designates the competition between the nucleation and the propagation of magnetic domains and $\beta$ a fractional exponent between 1 and 3 .

From the relation (10) we deduce another expression of $t_{1 / 2}$ :

$$
t_{1 / 2} \approx \frac{\tau(k)(\ln 2)^{\frac{1}{\beta}}}{R_{O}} \exp \left(\frac{\overline{\mathrm{E}_{\mathrm{a}}}-\mathrm{M}_{\mathrm{S}} \mathrm{V}_{\mathrm{B}}\left(\mu_{0} \mathrm{H}\right)}{\mathrm{K}_{\mathrm{B}} \mathrm{T}}\right)
$$


Hence by identification of Eq.(11) with Eq.(1), we get the prefactor as $t_{0} \cong \frac{\tau(k)(\ln 2)^{1 / \beta}}{R_{o}}$. Using this expression of $t_{0}$ with the values of its parameters measured [11] and recorded in table.3:

Table 3. Data of the parameters of magnetization reversal compressed exponential form

\begin{tabular}{|c|c|c|c|}
\hline $\mathbf{t}_{\mathbf{C}_{\mathbf{o}}}(\mathbf{n m})$ & $\mathbf{0 . 7}$ & $\mathbf{0 . 8}$ & $\mathbf{1}$ \\
\hline $\boldsymbol{\beta}$ & 1.41 & 1.73 & 1.49 \\
\hline $\mathbf{R}_{\mathbf{o}}\left(\mathbf{S}^{\mathbf{- 1}}\right)$ & $2 \times 10^{-5}$ & $3 \times 10^{-5}$ & $8.63 \times 10^{-6}$ \\
\hline $\boldsymbol{\tau}(\mathbf{k})$ & $5.5 \times 10^{-4}$ & $8.7 \times 10^{-4}$ & $2.4 \times 10^{-4}$ \\
\hline
\end{tabular}

We obtain the values of the width at zero field $\sigma_{w_{0}}$ recorded in the table .4

Table 4. Values of the Width of the distribution of energy barriers in each

\begin{tabular}{|c|c|c|c|}
\hline$t_{C_{o}}(\mathbf{n m})$ & $0.7 \mathrm{~nm}$ & $0.8 \mathrm{~nm}$ & $1 \mathrm{~nm}$ \\
\hline$\sigma_{w_{0}}(m e V)$ & 435.01 & 799.84 & 420.63 \\
\hline
\end{tabular}

We notice that these values are in perfect agreement with the values found by linear fitting in the $1 \mathrm{~nm}$ thick layer and are low compared to that found for in multilayers $(\mathrm{PtCo})_{3}$ [6]. And that the value of the width of the distribution of the energy barrier, when no field is applied, is generally low in all thicknesses and the lowest value is obtained in this thickness of $1 \mathrm{~nm}$ as shown fig.3. This means that it is easier to reverse the magnetization in the thickness of $1 \mathrm{~nm}$ than $0.7 \mathrm{~nm}$ and $0,8 \mathrm{~nm}$; which makes it possible to save energy in the latter. The high value within $0.8 \mathrm{~nm}$ can be explained by a high defect rate in this sample. These defects slow down the magnetization reversal process [10] ; hence this increases the width of the distribution of the energy barries in the sample.

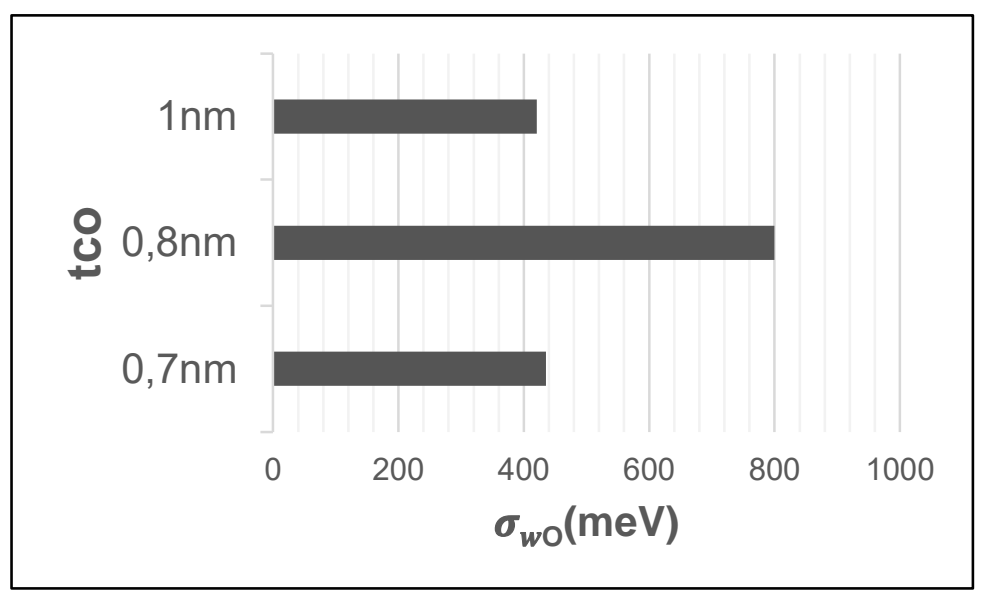

Figure 3. Width of the distributionof the energy barriers in each sample of thickness $\boldsymbol{t}_{\boldsymbol{C}_{o}}$ 


\section{Activation Energy barries in a real sample}

Activation fields in a real sample is the field $\mu_{0} \mathrm{H}_{\mathrm{r}}$ that must be applied to completely remove the width of the energy barrier distribution, i.e. to cancel the width of the barrier in a sample. From this definition we can then write :

$$
\sigma_{w_{0}}-2 \mu_{0} M_{S} V_{B} H_{r}=0
$$

We find respectively $\mu_{0} \mathrm{H}_{\mathrm{r}}=16.19 \mathrm{mT}, \mu_{0} \mathrm{H}_{\mathrm{r}}=15.80 \mathrm{mT}$ and $\mu_{0} \mathrm{H}_{\mathrm{r}}=10.04 \mathrm{mT}$ for the thickness $\mathrm{t}_{\mathrm{C}_{\mathrm{o}}}=0.7 \mathrm{~nm}, 0.8 \mathrm{~nm}$ and $1 \mathrm{~nm}$. These values compared to those of the coersive field $\mu_{0} \mathrm{H}_{\mathrm{c}}$ in table 1., are lower in the three cases, what shows that the magnetization reversal is well initiate in the real sample before it is demagnetized. The lowest value of $\mu_{0} \mathrm{H}_{\mathrm{r}}$ is found in the sample thickness $\mathrm{t}_{\mathrm{C}_{\mathrm{o}}}=1 \mathrm{~nm}$. It shows that magnetization reversal (the writing of elementary information) would not need enough electrical field ; therefore this sample will need less electrical energy.

\section{Distribution of energy barries}

The distribution of energy barries can be framed as follows [13]:

$$
\bar{E}_{a}-\frac{\sigma_{\mathrm{w}_{0}}}{2} \leq E_{a} \leq \bar{E}_{a}+\frac{\sigma_{\mathrm{w}_{0}}}{2}
$$

where $\bar{E}_{a}$ is the average activation energy in the sample, assumed to be equal to that found in table.2 . Using the Eq.(13) and Eq.(10), we get the following relation :

$$
-\mathrm{K}_{\mathrm{B}} \operatorname{Tlnt}_{0} \leq \mathrm{E}_{\mathrm{a}} \leq 2 \times \overline{\mathrm{E}}_{\mathrm{a}}+\mathrm{K}_{\mathrm{B}} \mathrm{Tlnt}_{0}
$$

On Fig 4. We show the distribution graph of the energy barrier in each sample.

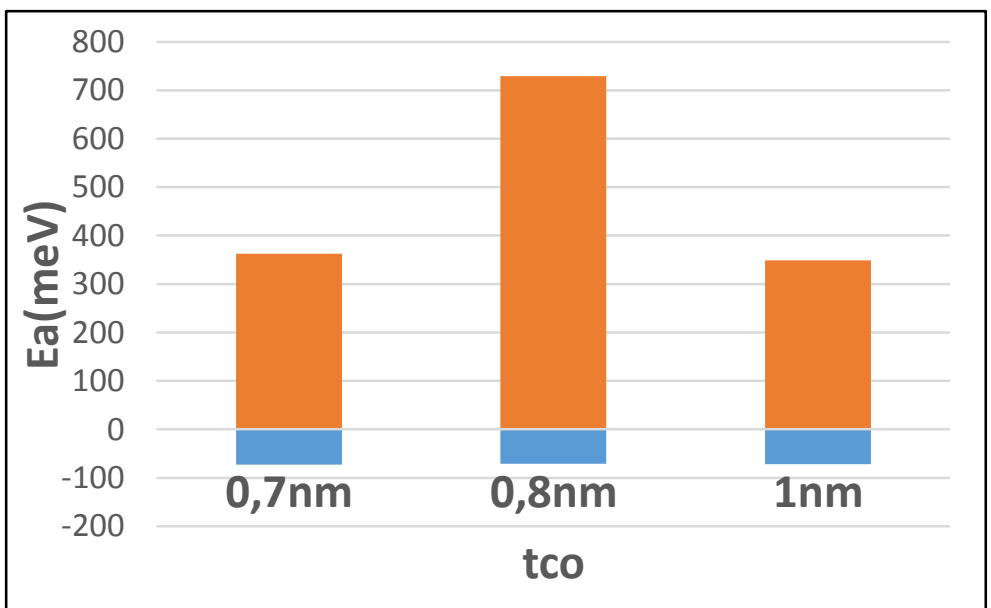

Figure 4. Distribution graph of the energy barrier in each sample

We find respectively the margins : $-72.38 \mathrm{meV} \leq E_{a} \leq 362,62 \mathrm{meV},-70,72 \leq E_{a} \leq$ 729.12 et $-71.91 \leq E_{a} \leq 348.71$ for thicknesses $\mathrm{t}_{\mathrm{C}_{\mathrm{o}}}=0.7 \mathrm{~nm}, 0.8 \mathrm{~nm}, 1 \mathrm{~nm}$. It is clear here 
too that the distribution margin of the energy barrier in the sample of thickness $t_{C_{o}}=1 \mathrm{~nm}$ is smaller. This shows that reversing the magnetization in the sample $t_{\mathrm{C}_{o}}=1 \mathrm{~nm}$ will require less electrical energy ; so less electrical energy for information storage.

\section{Conclusion}

We have shown through this article the effect of the thickness of the magnetic layers of cobalt on the distribution of the energy barrier and therefore on the saving of electrical energy in the storage of information in these media. We theoretically calculated, the distribution of the energy barrier $\mathrm{E}_{\mathrm{a}}$, its distribution width $\sigma_{w}$ and the real activation field $\mu_{0} \mathrm{H}_{\mathrm{r}}$, and saw that all are different and weak in each thickness $\mathrm{t}_{\mathrm{C}_{\mathrm{o}}}=0.7 \mathrm{~nm}, 0.8 \mathrm{~nm}$ and $1 \mathrm{~nm}$. Moreover, the smallest value of these parameters are found in the layer of thickness $\mathrm{t}_{\mathrm{C}_{\mathrm{o}}}=1 \mathrm{~nm}$. Therefore, this state that the writing a bit of information in this thickness of $1 \mathrm{~nm}$ would require less magnetic field. Hence the storage of information in cobalt thickness of $1 \mathrm{~nm}$ would require less electrical energy.

\section{References}

[1] Pommier J, Meyer P, Pénissard G, Ferré J, Bruno P, Renard D. Magnetization reversal in ultrathin ferromagnetic films with perpendicular anistropy: Domain observations. Physical Review Letters. 1990 Oct 15;65(16):2054-2057. https://doi.org/10.1103/physrevlett.65.2054

[2] Shen JX, Kirby RD, Wierman K, Shan Z-, Sellmyer DJ, Suzuki T. Magnetization reversal and defects in Co/Pt multilayers. Journal of Applied Physics. 199305 15;73(10):6418-6420. https://doi.org/10.1063/1.352618

[3] Bruno P, Bayreuther G, Beauvillain P, Chappert C, Lugert G, Renard D, Renard JP, Seiden J. Hysteresis properties of ultrathin ferromagnetic films. Journal of Applied Physics. 1990 Dec;68(11):5759-5766. https://doi.org/10.1063/1.346944

[4] Adanlété Adjanoh A, Belhi R. Energy Depending on the Thickness of the Ferromagnetic Layer. J. Mat. Phys and Chem. 2018;.

[5] Czapkiewicz M, Stobiecki S, van Dijken. Thermally activated magnetization reversal in exchange-biased [PtCo]3/Pt//rMn multilayers. Phys. Rev. B. 2008;77.

[6] Belhi R, Adanlété Adjanoh A, Vogel J. Influence of Pt thickness on magnetization reversal processes in (Pt/Co)3 multilayers with perpendicular anisotropy. Journal of Magnetism and Magnetic Materials. 2012 06;324(11):1869-1877. https://doi.org/10.1016/j.jmmm.2012.01.003

[7] Chappert C, Renard P, Beauvillain J. Ferromagnetism of very thin films of nickel and cobalt. J. Magn. Magn. Mater. 1986;54:795.

[8] Lee CH, He H, Lamelas F, Vavra W, Uher C, Clarke R. Epitaxial Co-Au Superlattices. Physical Review Letters. 198902 06;62(6):653-656.

https://doi.org/10.1103/physrevlett.62.653

[9] Ohtake M, Futamoto M, Kirino F, Fujita N, Inaba N. Epitaxial growth of hcp/fcc Co bilayer films on Al2O3(0001) substrates. Journal of Applied Physics. 2008 04;103(7):07B522. https://doi.org/10.1063/1.2838848

[10] Adjanoh AA, Belhi R. Micro magnetic dendritic domain structure in the demagnetized state of perpendicular magnetic ultrathin film: The effect of the width of the distribution of the energy barriers. Journal of Magnetism and Magnetic Materials. 2019 03;473:12-15. https://doi.org/10.1016/j.jmmm.2018.10.025

[11] Adanlété Adjanoh A, Belhi R, Vogel J, Ayadi M, Abdelmoula K. Compressed exponential form for disordered domain wall motion in ultra-thin Au/Co/Au ferromagnetic films. Journal of 
Magnetism and Magnetic Materials. 2011 03;323(5):504-508.

https://doi.org/10.1016/j.jmmm.2010.10.002 